\title{
SPECTROSCOPY OF EXOTIC BARYONS WITH CLAS: SEARCH FOR GROUND AND FIRST EXCITED STATES
}

\author{
M. BATTAGLIERI \\ Istituto Nazionale di Fisica Nucleare, \\ Via Dodecaneso 33 \\ Genova, 16100, Italy \\ E-mail:battaglieri@ge.infn.it
}

\begin{abstract}
In the las year many Collaborations reported about the evidence of a possible pentaquark state but so far the results are not yet conclusive. New dedicated experiments with higher statistics and precision are necessary to confirm the pentaquark existence and its properties. In this contribution I will report about a new photoproduction experiment on a proton target, the so called 'G11' experiment, just performed in the Hall-B at Jefferson Lab that collected ten times the existing statistics.
\end{abstract}

\section{Introduction}

The first evidence of a narrow resonance with $S=+1$, named $\Theta^{+}$, was reported by the LEPS Collaboration ${ }^{1}$ and then confirmed by other experimental groups ${ }^{2,3,4,5,6}$ including the CLAS Collaboration who found a signal in the reactions $\gamma D \rightarrow p K^{+} K^{-}(n)^{7}$ and $\gamma p \rightarrow \pi^{+} K^{-} K^{+}(n){ }^{8}$. However, due to the limited statistics, the reported results are not yet conclusive and experiments with higher statistics are needed to confirm these findings and do extensive checks of the systematic dependencies. Jefferson Lab and, in particular, the CLAS Collaboration in the Hall-B started a comprehensive experimental program ${ }^{9}$ to establish a firm and consistent phenomenology of the $\Theta^{+}$spectra, to determine in which production and decay channels the $\Theta^{+}$is seen, and what the production mechanisms are. The G11 experiment run for 65 calendar days in June-July 2004 using the CLAS detector and the Hall-B Bremsstrahlung photon beam on a proton target. We measured two production channels, $\gamma p \rightarrow \bar{K}^{0} \Theta^{+}$and $\gamma p \rightarrow K^{*} \Theta^{+}$, as well as two decay modes of the $\Theta^{+}, K^{+} n$ and $K^{0} p$ hoping to answer the following questions:

- are the present signals a statistical fluctuation? 
- in which production and decay channels does the $\Theta^{+}$show up?

- what is the angular dependence of the associated $K$ meson?

- what are the production mechanisms in terms of hadron dynamics?

- what are the relative production cross-sections and branching ratios?

and, most importantly,

-what are the masses and widths of pentaquarks produced in photoproduction and decaying to a $K$-nucleon final state? This measurement will provide a solid foundation for a long-term plan for the investigation of the pentaquark spectrum and properties.

\section{Theoretical predictions}

Theoretical predictions on the existence and properties of pentaquark baryons are based on a variety of models that treat the basic degrees of freedom quite differently. Due to the lack of a well-established phenomenology, at present different approaches result in different expectations for masses, widths, and quantum numbers of the $\Theta^{+}$and its companions. A discussion of different scenarios for pentaquark spectroscopy is found in the paper by Close ${ }^{10}$, where it is remarked that a spin-3/2 partner of the $\Theta^{+}$may exist. The predicted mass gap varies from $50 \mathrm{MeV}$, obtained in the Skyrmesoliton model by Borisuyk and collaborators ${ }^{11}$, to $250 \mathrm{MeV}$, reported in Ref. ${ }^{12}$. Very recently, a general classification of all the possible pentaquark states based on symmetry considerations has been reported in ${ }^{13}$. From this model-independent discussion, it emerges that there should be a pair of states with spin $1 / 2$ and $3 / 2$, respectively, with a splitting due to a spinorbit interaction in the positive parity case, or to a spin-spin interaction in the negative parity case. The ground state is expected to have $\mathrm{I}=0$ while the first excited should be an iso-triplet. Estimates of the total and differential cross sections for the reactions $\gamma p \rightarrow \bar{K}^{0} \Theta^{+}$have been carried out in hadronic models with effective Lagrangians ${ }^{14}$ and in the Regge theory approach. At present, the production mechanisms are completely unknown and the available calculations include contributions from meson-exchange in the $t$-channel, baryon exchange in the $s$-channel, and pentaquark exchange in the $u$-channel. Varying the unknown coupling constants within reasonable ranges, such models are able to predict the total and differential cross sections according to different hypotheses for the $\Theta^{+}$quantum numbers (parity, spin, isospin, etc.). The result of the G11 experiment will help in constraining the model predictions. 


\section{Present CLAS measurements}

Several photon-induced reactions have been studied using the available data on a proton target collected by CLAS in previous years.

In the energy range 1.8-3.0 GeV we analyzed the reaction $\gamma p \rightarrow \bar{K}^{0} \Theta^{+}$ and subsequent $\Theta^{+}$decay to $K^{+} n$ or $K^{0} p$. We studied the two final states:

$\gamma p \rightarrow \bar{K}^{0} \Theta^{+} \rightarrow \pi^{+} \pi^{-} K^{+}(n)$

$\gamma p \rightarrow \bar{K}^{0} \Theta^{+} \rightarrow \pi^{+} \pi^{-} p\left(K^{0}\right)$

The $\bar{K}^{0}\left(K^{0}\right)$ was identified by detecting its $K_{s}^{0}$ component decaying to $\pi^{+} \pi^{-}$(b.r. $\sim 68.6 \%$ ). For both channels three charged particles in final states were required: $\pi^{+} \pi^{-} K^{+}$in the first case and $\pi^{+} \pi^{-} p$ in the second case. The neutron (or $K^{0} / \bar{K}^{0}$ ) was identified using the missing mass technique. In the former reaction, the $\Theta^{+}$should show up in the $\bar{K}^{0}$ missing mass while in the latter it should be seen both in the detected $K^{0}$ missing mass or in the $\left(K^{0} p\right)$ system invariant mass. After removing the background coming from hyperon production with the same final state $\left(K^{+} \Lambda^{*}(1520)\right.$, $\left.K^{+} \Sigma^{+(-)} \pi^{-(+)}\right)$, the $\left(K^{+} n\right)$ and $\left(K^{0} p\right)$ invariant mass spectra showed two possible narrow structures that were enhanced by selecting the low energy region $\left(1.8<E_{\gamma}<2.3 \mathrm{GeV}\right)$, close to the expected production threshold for the $\Theta^{+}$. Although this analysis is still in a preliminary stage and parallel analyses on the same data set show that statistical fluctuations using different selection cuts are not negligible, it calls for a new experiment with high statistics and precision,

At higher energy, 3.0-5.0 GeV, we studied the reactions $\gamma p \rightarrow \bar{K}^{0} \Theta^{+} \rightarrow$ $\bar{K}^{0} K^{+} n$ and $\gamma p \rightarrow K^{*} \Theta^{+} \rightarrow K^{-} \pi^{+} K^{+} n$. In all of these channels we found possible evidence of a narrow peak in the $\left(K^{+} n\right)$ invariant mass located in the range $1.55-1.57 \mathrm{GeV}$. The results of the $\gamma p \rightarrow K^{*} \Theta^{+}$channel have been published in $\mathrm{PRL}^{8}$.

\section{The experimental set-up}

G11 experiment measures two production channels on the proton: $K^{0} \Theta^{+}$ and $K^{*} \Theta^{+}$, each using two decay modes of the $\Theta^{+}: K^{+} n$ and $K^{0} p$, for a total of four final states. The primary goal of the experiment is to establish the mass spectra with a precise measurement of the masses, widths, and errors on any peaks observed. With the high statistics collected in the running time, a determination of the total and differential cross sections as well as the decay angular distribution will also be possible.

The experiment uses the Hall B Bremsstrahlung tagged photon beam and the CLAS detector with a $40 \mathrm{~cm}$ hydrogen target. The primary electron 


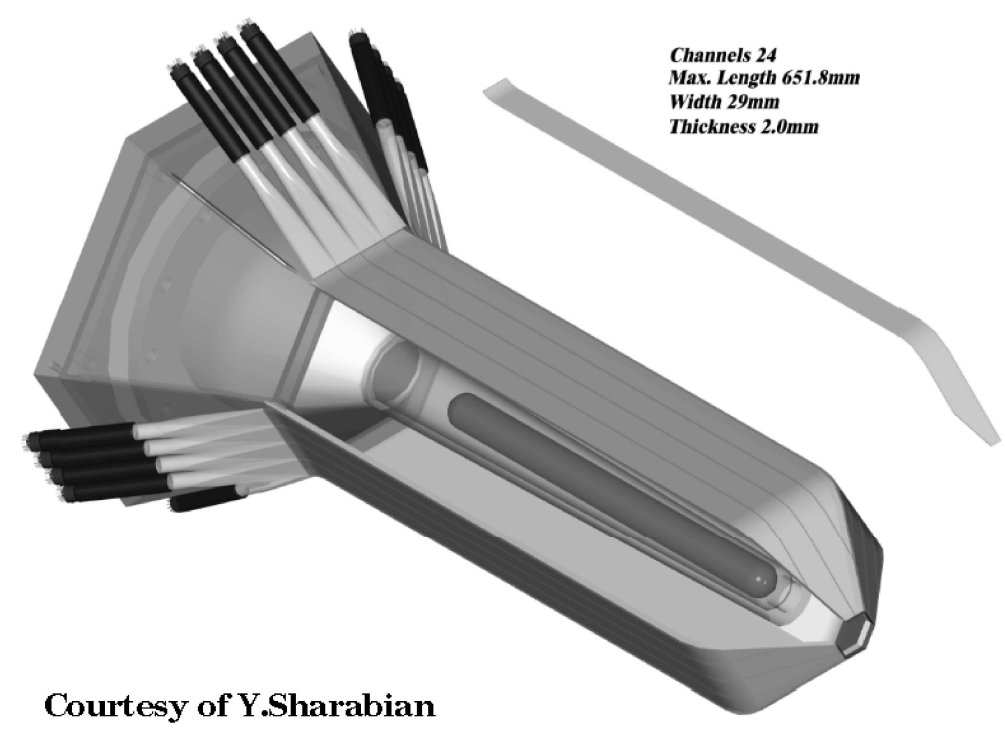

Figure 1. The new CLAS Start Counter used for the first time in G11 experiment.

beam has an energy of $4 \mathrm{GeV}$ to obtain tagged photons from the $\Theta^{+}$production threshold $(1.6 \mathrm{GeV})$ to the maximum energy of $3.8 \mathrm{GeV}$. The CLAS spectrometer ${ }^{15}$ (CEBAF Large Acceptance Spectrometer), is built around six superconducting coils producing a toroidal magnetic field. The detector package consists of three layers of drift chambers for track reconstruction, one layer of scintillators for time-of-flight measurements and hadron identification, forward Cerenkov counters for electron-pion discrimination, and electromagnetic calorimeters to identify electrons and neutral particles. It is well suited for simultaneous multi-hadron detection as required by the pentaquark experiments. Using CLAS together the Hall-B tagger facility, we will be able to study simultaneously the quoted reactions with a high experimental resolution. The experimental set-up (polarity and strength of the CLAS magnetic field, beam energy, trigger condition) was optimized by performing full $\mathrm{MC}$ simulations and exploiting the experience gained analyzing the existing CLAS data.

A new longer start counter was specifically built to trigger the data acquisition. The new CLAS start counter is made by 24 strips of $2 \mathrm{~mm}$ thick plastic scintillator with a single side PMT-based read-out. A time resolution of few ns, reduced to 300ps in the off-line analysis was achieved. 
The high azimuthal segmentation allows to work with an higher photon flux (around $60 \mathrm{MHz}$ on the tagger focal plane) reducing the time necessary to collect ten times the existing statistics to a reasonable number of days ( $\sim 30$ PAC days). A drawing of the CLAS new start counter is shown in fig. 1
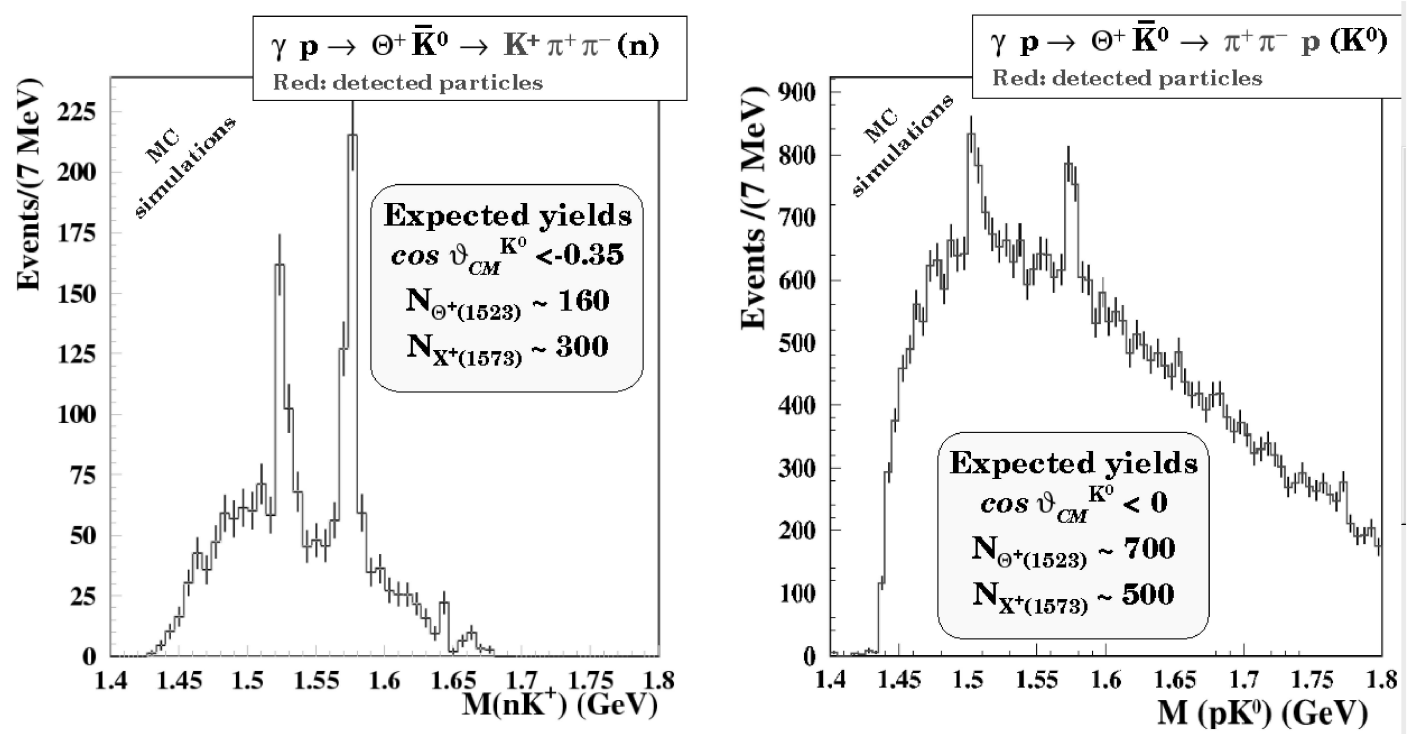

Figure 2. The $\left(K^{+} n\right)$ (left) and $p K^{0}$ (right) invariant mass spectra for $K^{0}$ at backward angles in the center-of-mass system.

\section{Expected statistical accuracy}

The statistical accuracy of G11 experiment was estimated by extrapolating the results obtained from the low-energy CLAS data analysis. The projected yields reported below refer to the photon energy range $E_{\gamma}=1.8-2.3$ $\mathrm{GeV}$ close to the $\Theta^{+}$production threshold (where the production cross section should be maximum) therefore represent a lower limit of the achievable statistics. Results are shown for the reactions $\gamma p \rightarrow \bar{K}^{0} \Theta^{+} \rightarrow \pi^{+} \pi^{-} K^{+}(n)$ and $\gamma p \rightarrow \bar{K}^{0} \Theta^{+} \rightarrow \pi^{+} \pi^{-} p\left(K^{0}\right)$ with the CLAS magnetic field to $50 \%$ of its maximum, assuming a production cross section of $16 \mathrm{nb}$, equal branching ratios for $\Theta^{+}$decaying to $n K^{+}$and $p K^{0}$, and an integrated luminosity corresponding to 25 days/run. We expect $160(700) \Theta^{+}(1526)$ and $300(500)$ 
$\Theta^{+}(1571)$ candidates for the $n K^{+}\left(p K^{0}\right)$ final state, cutting at backward angles. The expected $n K^{+}$and $p K^{0}$ invariant mass spectra are shown in Fig. 2.

With the same assumptions, the expected statistical error on the differential cross section has been estimated. Fig. 3 shows the expected results for the $\gamma p \rightarrow \bar{K}^{0} \Theta^{+} \rightarrow \pi^{+} \pi^{-} K^{+}(n)$ channel, according to different hypotheses on production mechanism: a flat distribution in the $\left(K^{0}-\Theta^{+}\right)$ center-of-mass, $u$-channel production, and $t$-channel production.

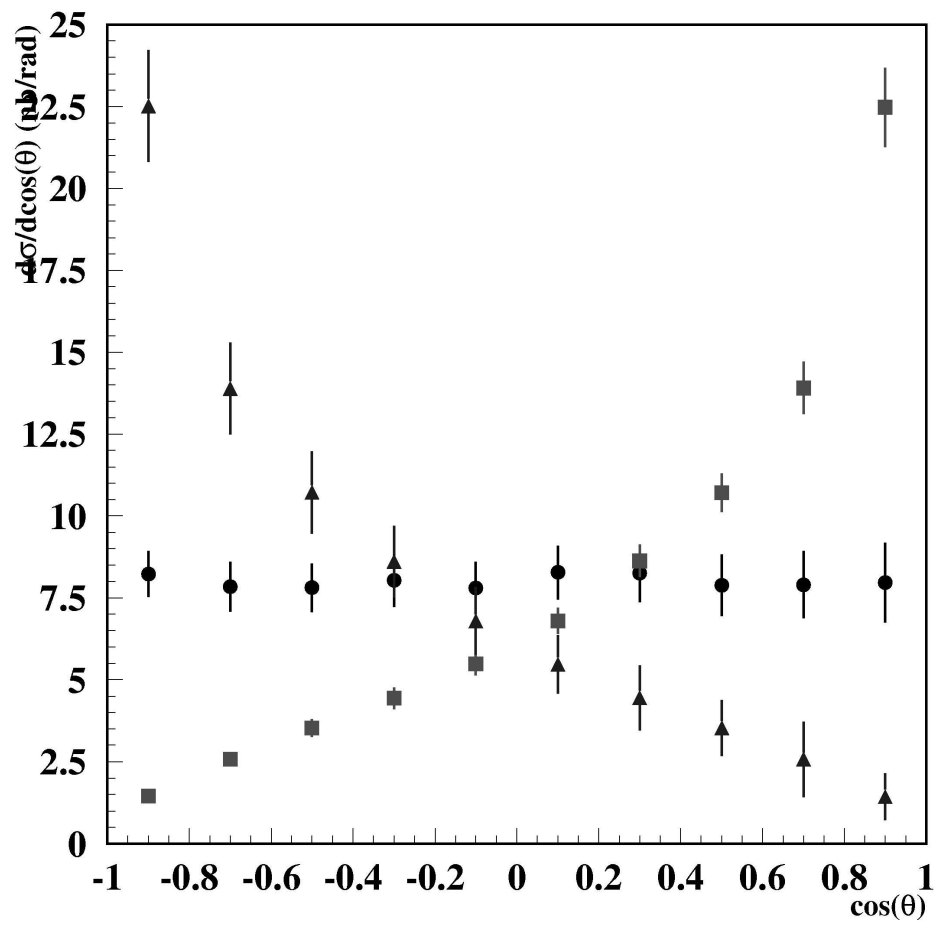

Figure 3. The expected errors on the differential cross section according to different production mechanisms: flat (dots), $u$-channel production (triangles), and $t$-channel (squares).

Decay angular distribution $(\mathrm{W}(\theta))$ reflects spin and parity of the $\Theta^{+}$. Different combinations of the two quantum numbers are related to different 
angular behavior (e.g. if $\mathrm{J}^{P}=1 / 2^{+}$and $\mathrm{J}_{z}= \pm 1 / 2$ then $\mathrm{W}(\theta)=$ constant; if $\mathrm{J}^{P}=3 / 2^{+}$and $\mathrm{J}_{z}= \pm 1 / 2$ then $\mathrm{W}(\theta)=1+3 \cos ^{2} \theta$ while if $\mathrm{J}_{z}= \pm 3 / 2$ then $\left.\mathrm{W}(\theta)=\sin ^{2} \theta\right)$. If the $\Theta^{+}$is produced in some defined polarization state, measuring the decay angular distribution will provide some informations about them. The G11, will measure the decay angular distributions with the statistics accuracy shown in Fig. 4.

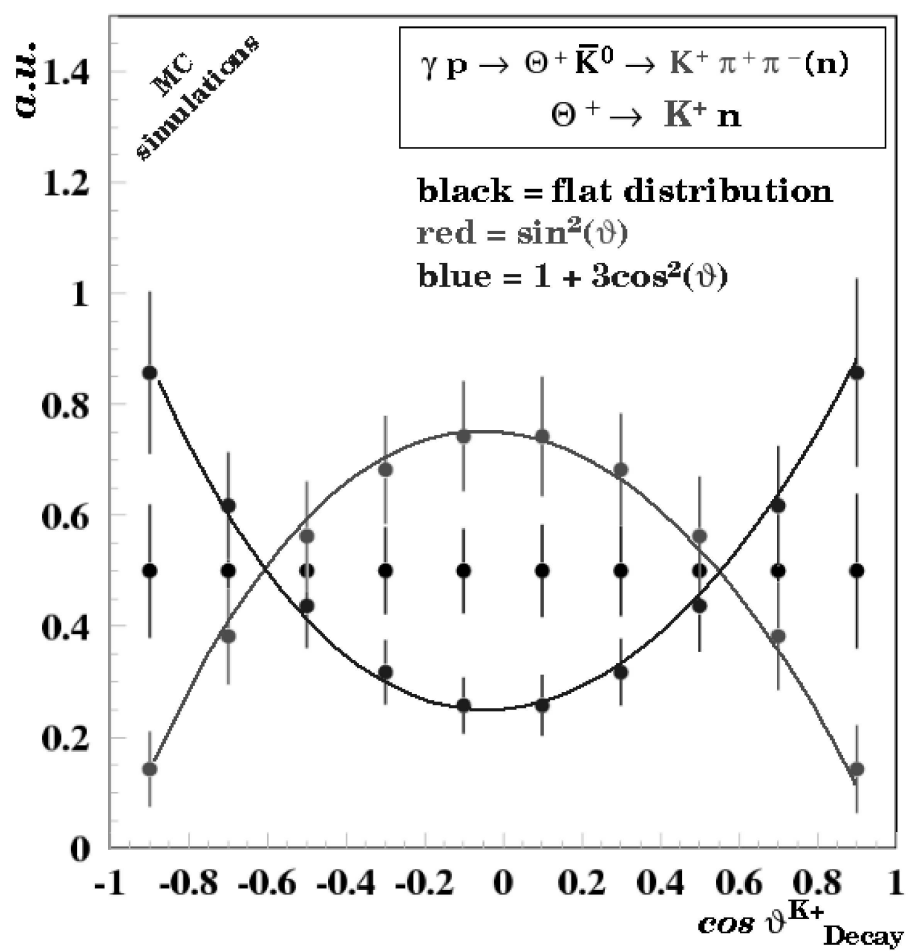

Figure 4. The espected error on the $\Theta^{+}$decay distributions according to different combination of spin and parity.

\section{Summary}

Experimental evidence for a pentaquark state of baryonic matter is increasingly convincing. However, many small contradictions plague the comparison of experimental results. No single experiment has the statistical power to rule out the chance of a correlation between a statistical fluctuation and 
an unknown systematic enhancement acting to produce the state. The G11 experiment at Jefferson Lab will firmly establishing the phenomenology of the $\Theta^{+}$spectrum. Data were taken in June-July 2004 and now they are under analysis. With ten times the statistics of our present data sample, this new experiment will enable us to pin down the masses and widths of any peaks in the spectrum and measure differential and total cross section helping to determine the relevance of pentaquark production mechanisms.

\section{References}

1. T. Nakano et al., Phys. Rev. Lett. 91, 012002 (2003).

2. V.V. Barmin et al., Phys. Atom. Nucl. 66, 1715 (2003); Yad. Fiz. 66, 1763 (2003).

3. J. Barth et al., Phys. Lett. B 572, 127 (2003).

4. A.E. Asratyan, A.G. Dolgolenko, and M.A. Kubantsev, Phys. Atom. Nucl. 67, 682 (2004); Yad. Fiz. 67, 704 (2004).

5. A. Airapetian et al., Phys. Lett. B585 213, (2004).

6. S. Chekanov et al., Phys. Lett. B591 7, (2004).

7. S. Stepanyan et al., Phys. Rev. Lett. 91, 252001 (2003).

8. V. Kubarovsky et al.,Phys. Rev. Lett. 92, 032001 (2003).

9. M. Battaglieri et al., JLab Experiment 04-021.

10. F. Close and J.J. Dudek, Phys. Lett. B586 75, 2004.

11. D. Borisyuk, M. Faber and A. Kobushkin, hep-ph/0307370.

12. B.K. Jennings and K. Maltman, Phys. Rev. D69, 094020, 2004.

13. R. Bijker, M. M. Giannini and E. Santopinto, hep-ph/0310281.

14. Y. Oh et al., Phys. Rev. D69, 014009, 2004.

15. B. Mecking et al.,Nucl. Instrum. and Meth. A503, 513 (2003). 